\title{
POBLADOS DE ALTURA DE LA EDAD DEL BRONCE EN LAS SIERRAS DE PICON Y PIEDRABUENA (CIUDAD REAL). CONTRIBUCION AL ANALISIS ESPACIAL DEL TERRITORIO
}

\author{
POR \\ FRANCISCO JAVIER LOPEZ FERNANDEZ (*)
}

\begin{abstract}
RESUMEN En este artículo se lleva a cabo la revisión de una serie de asentamientos, que tradicionalmente se venian adscribiendo a la Cultura Celta, utilizando para ello los planteamientos propugnados por la Arqueología Espacial de conocimiento y análisis del yacimiento y su entorno. También se incluyen una serie de asentamientos descubiertos durante la realización de los trabajos de campo.

Del análisis global de sus características se desprende que estos «enclaves arqueológicos» deben ser incluidos en el denominado Bronce de la Mancha.

SUMMARY In this paper we will revise a series of settlements traditionally adscribed to the Celtic Culture. For that purpose we will use the viewpoint of knowledge and site analysis and its environment defended by the Spatial Archeology. A series of sites discovered during the field work is also included. From the global analysis of their characteristics it can be understood that these archeological sites must be dated in the Bronze Age of La Mancha.
\end{abstract}

Palabras clave Yacimientos de Altura, Arqueología Espacial, Sierras Marginales, Modelos de Ocupación, Collado, Cima, Ladera, Bronce Manchego.

El presente artículo es un resumen de un trabajo más extenso presentado para los cursos de doctorado en la Universidad Autonóma de Madrid y tiene su origen en una serie de estudios arqueológicos llevados a cabo por distintos investigadores (Hierro, 1973; Caballero, 1983; López, 1988) sobre diversos asentamientos prehistóricos de altura (castellones) en la provincia de Ciudad Real y cuyas opiniones sobre algunos de estos «lugares" eran netamente contradictorias, llegando algunos de ellos a identificarlos como pertenecientes a la cultura castreña celta. Por ello decidimos llevar a cabo una revisión del tema, aplicando las técnicas de análisis del territorio desde el punto

(") Personal Laboral del Ministerio de Cultura, adscrito al Museo Provincial de Ciudad Real, C/ Calatrava, n 10, 2." 3." 13004 Ciudad Real. 
de vista de la Arqueología Espacial, con el fin de obtener el mayor número de datos posible para de este modo llegar, al menos, a una identificación cultural.

Un primer análisis de los datos obtenidos dio como resultado algunas diferencias con respecto a las afirmaciones de nuestros predecesores en el tema, sobre todo en lo que a materiales arqueológicos se refiere, pues ninguno de los autores citados habia localizado restos materiales (cerámica o sílex), en cambio nosotros recogimos alguno en la mayor parte de los yacimientos prospectados. Por este motivo pensamos que podría ser positivo el profundizar en las notas tomadas a pie de campo para ver cuáles podian ser los resultados finales, máxime teniendo en cuenta que los patrones de asentamiento eran muy similares a los que estábamos observando en zonas próximas para asentamientos pertenecientes a la Edad del Bronce.

El área objeto de nuestro estudio se encuentra situada, geográficamente, entre los términos municipales de Picón y Piedrabuena, provincia de Ciudad Real, en una serie de sierras marginales de los Montes de Toledo, cuya característica común en su altura (700-850 mts. sobre el nivel del mar). La superficie que vamos a analizar se corresponde plenamente con la que, en su día, estudió Hierro del Real (Hierro, 1973) y por ello aparecen algunas lagunas de poblamiento en la zona, que sin duda quedarán cubiertas cuando finalicen los trabajos que estamos llevando a cabo en la zona norte de la provincia de Ciudad Real.

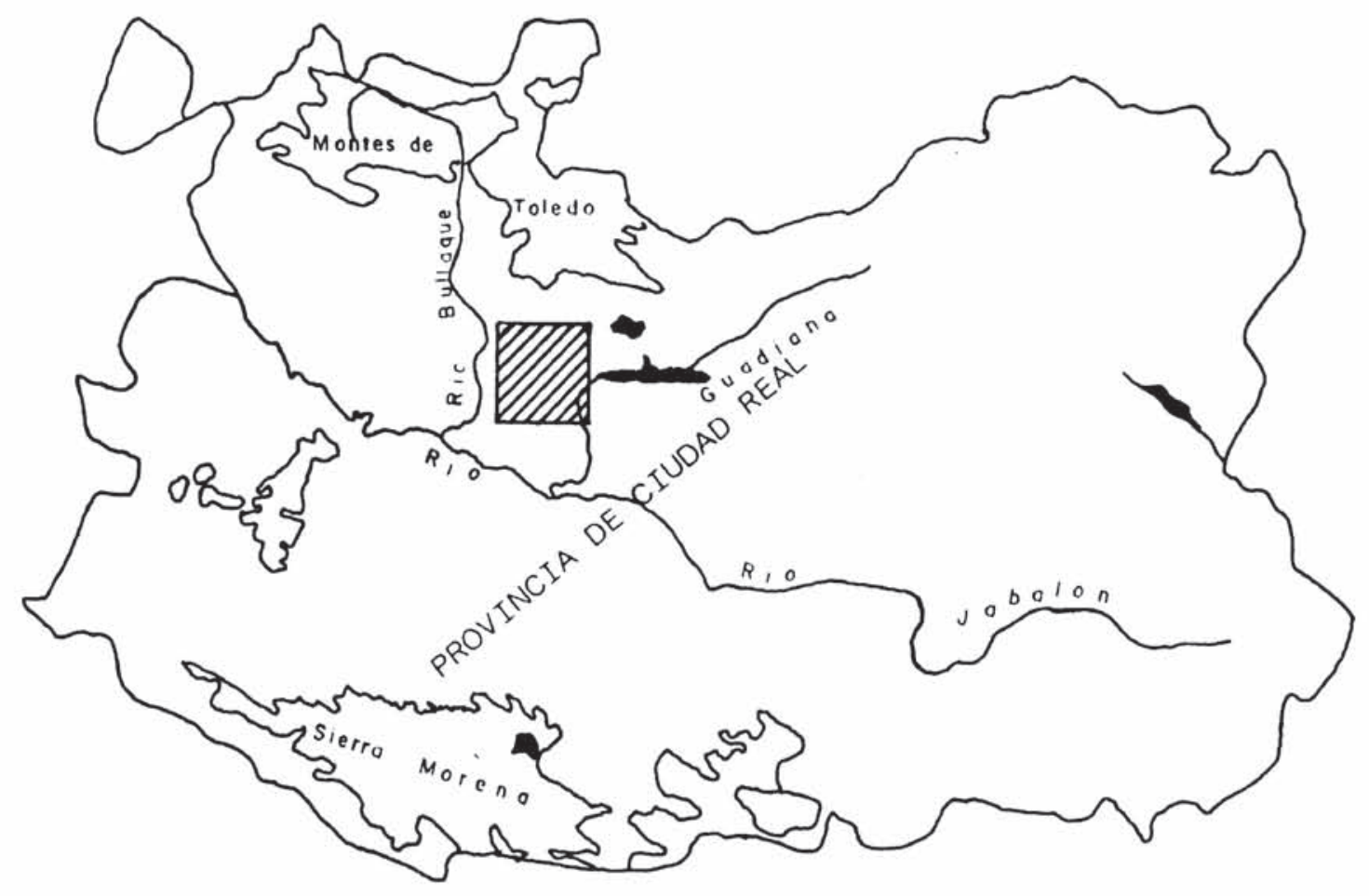

Fig. 1. Mapa de situación del área en estudio.

El proceso de localización y estudio de los yacimientos arqueológicos que hemos seguido pasa por dos fases, que se complementan entre sí.

En la primera se trataba de localizar los asentamientos y para ello utilizamos la fotografía aérea, el análisis cartográfico, la encuesta directa, la consulta bibliográfica (Viñas, 1971; Hierro, 1973; Caballero, 1983): dando por concluida esta fase con la prospección exhaustiva de la zona en estudio.

La labor más difícil de llevar a cabo fue el reconocimiento visual del terreno, que se realizó dividiendo las sierras en tres zonas (solana, cumbre y umbría), tarea que corrió a cargo de un 
equipo formado por tres personas. A la localización de cada vacimiento correspondia el registro del mismo, empleando para ello las fichas utilizadas por el equipo de la Universidad Autónoma (Miranda, 1986) que desde hace algunos años investiga sobre la Edad del Bronce en la Mancha.

En la segunda fase debiamos estudiar los distintos datos recogidos, teniendo en cuenta aspectos tales como la organización espacial del entorno, rasgos definitorios del asentamiento (ubicación, arquitectura, materiales, etc.), así como los derivados de las relaciones entre distintas colectividades (visibilidad, territorio de explotación, comunicaciones, etc.).

Los yacimientos del área de Picón y Piedrabuena se encuentran ubicados en una serie de sierras marginales (Sur) de los Montes de Toledo. Estas sierras separan las cuencas de los ríos Guadiana y Bullaque, al mismo tiempo que sirven como frontera natural al Campo de Calatrava, sobre cuyas planicies ejercían amplio control visual los pobladores que en estas cumbres se asentaron.

El grupo o grupos humanos localizados en este área sitúan sus asentamientos en las cumbres y laderas de las sierras, buscando un lugar idóneo para el emplazamiento de las viviendas. Estos «sitios" se caracterizan por tener una fácil defensa, un amplio dominio visual sobre el entorno y un cómodo acceso a los recursos. Estas constantes también hemos podido observarlas en los Montes de Toledo (López, 1988) por lo que la diversidad de emplazamientos parece querer indicarnos que nos hallamos ante "modelos de ocupación" preestablecidos, máxime si tenemos en cuenta que en todos los casos analizados los terrenos óptimos para la explotación agrícola se encuentran a una distancia que oscila entre 1 y 1,5 kms., hallándose dentro del marco considerado como "territorio ideal de explotación" (Chisholm, 1968; Lee, 1969).

Para la ubicación del poblamiento se buscan aquellos lugares donde afloran las cuarcitas, en cuyos espacios libres se distribuye el habitat, utilizando las paredes rocosas como apoyo de las viviendas, sirviendo de este modo como parapeto a los vientos casi huracanados que se producen en primavera y otoño. Por otro lado, estos farallones se utilizan como defensas naturales, aunque no por ello dejan de construirse potentes murallas, rellenando los espacios libres en la pared rocosa con hiladas de piedra, evitando, de este modo, el costo tan elevado (tiempo y mano de obra) que supondría el fortificar una superficie completamente llana.

En algunos de estos asentamientos el carácter estratégico está determinado por su situación en las proximidades de puertos o collados ( $\mathrm{n}$. os $1,5,7$, y 9), adquiriendo así, pleno dominio de las rutas comerciales y de las vías de comunicación (Fig. 2). Cabe señalar que esta zona ocuparía un lugar intermedio en las comunicaciones Norte-Sur (Andalucía-Meseta Norte, cruzando los Montes de Toledo por el paso de la Torre de Abraham y Puerto del Milagro en los Montes de Toledo) y EsteOeste (Levante-Extremadura, aprovechando los pasos naturales que se forman en la unión de los Montes de Toledo y una serie de sierras marginales, localizadas al Oeste de la provincia de Ciudad Real) por lo que las poblaciones de la zona habrian de encargarse del control de estas dos rutas y de los trazados alternativos (Yac. en Collado). Una de ellas nos parece especialmente importante (Norte-Sur), pues muy probablemente, sería utilizada para transportar el mineral de cobre que se extraía en la zona norte de los Montes de Toledo, en el actual término municipal de San Pablo de los Montes, en la provincia de Toledo.

En otros, en cambio, el matiz táctico viene definido por su posicionamiento sobre los puntos de mayor altura, en cotas por encima de los $770 \mathrm{~m}$. (Fig. 3) y condiciones más favorables para la defensa (carácter bélico), ejerciendo, al mismo tiempo, un amplio control visual del territorio, advirtiendo cualquier movimiento de personas o animales que se produjera en la llanura $(n . \underline{\text { s }} 3,6,8$, $10,12$ y 14$)$.

La localización de yacimientos por debajo de las cotas máximas (media altura) parece indicar que no todos los asentamientos tenian como principal cometido el control del territorio, pues los situados en ladera tienen una mejor ubicación de cara al cultivo y aprovechamiento de los valles y vaguadas próximos (tierras fértiles, aptas para el cultivo de cereal) y por tanto, una buena disposición para el cuidado y defensa de sus tierras de labor.

Atendiendo a las características de su emplazamiento, los yacimientos han sido clasificados en tres grandes grupos, aunque no por ello dejan de existir pequeñas matizaciones entre los componentes de cada uno de los tipos. Los modelos propuestos son los siguientes: 


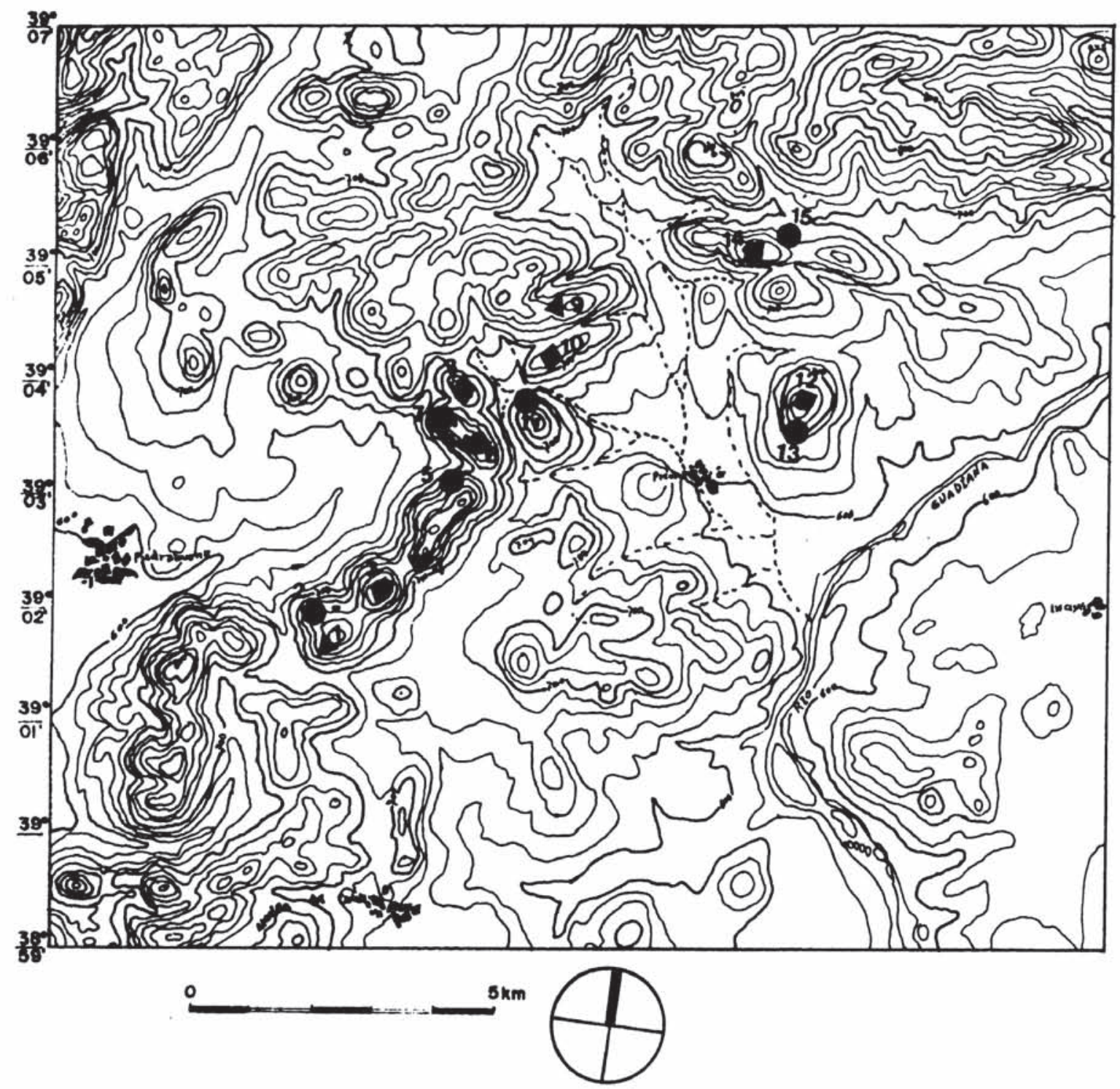

Fic. 2. Situación del área de estudio y ubicación de los yacimientos: Cima. $\Delta$ Collado. Ladera. 1) Morro el Sotajo. 2) Morro Pinto. 3) Cerro Benavente. 4) Collado de los Criminales. 5) Hoya del Lobo. 6) Aljibe la Vieja. 7) Morro de la Dueña. 8) Aljibe el Moro. 9) Cerro Cañejares. 10) Cerro el Tesoro. 11) Cerro Majadaclemente. 12) Plaza de los Moros. 13) Cerro de la Cantera. 14) Cerro del Acebuchar 1, 15 Cerro del Acebuchar II.

a) Yacimientos en collado ( $n .0$ s 1, 4 y 9). Estos se localizan en las proximidades de los pasos o puertos naturales (Fig. 2), en cotas que oscilan entre los 760-800 m. sobre el nivel del mar y a 160$200 \mathrm{~m}$. sobre el llano circundante (Fig. 3), a través de los cuales resultan fáciles las comunicaciones entre los valles de Picón y Piedrabuena.

Estos asentamientos tienen un fácil acceso a los recursos del entorno, agrícolas, ganaderos, de recolección, caza, etc., aunque cabe suponer que una buena parte de su actividad habría de estar relacionada con el control de los pasos naturales sobre los que se ubican.

b) Yacimiento en cima (n.오 3, 6, 8, 10, 12 y 14). Se localizan en puntos elevados de la sierra (Fig. 
2), en cotas entre los $750-838 \mathrm{~m}$. sobre el nivel del mar y alrededor de los $160-230 \mathrm{~m}$. sobre la llanura, aunque no siempre coincidiendo con las máximas alturas (Fig. 3). Su distribución a lo largo de la cuerda de las sierras permite un fácil control visual del territorio, que en ocasiones se prolonga a decenas de kms. (n.․ㅗ 3 y 12), enlazando con yacimientos de otras áreas.

c) Yacimientos en ladera ( $\mathrm{n}$. $\underline{2}$ 2, 5, 7, 11 y 15). Son aquellos que se encuentran emplazados en la falda de la sierra (Fig. 2), en pequeños promontorios o espolones rocosos, que se introducen en la llanura. Se localizan en cotas que oscilan entre los 740-750 m. sobre el nivel del mar y a $140-150 \mathrm{~m}$. sobre la llanura (Fig. 3). Estos asentamientos se definen, a nuestro modo de ver, por su buena disposición para el aprovechamiento de los fondos de valle, a los que pueden acceder fácilmente y por la subordinación a uno o varios de los pertenecientes a los grupos 3 y 4 , al menos en lo que al establecimiento de relaciones visuales se refiere.

Las diferencias de emplazamiento existentes entre unos yacimientos y otros nos llama poderosamente la atención, al mismo tiempo que nos inducen a considerar la posibilidad, por otro lado perfectamente factible, de una organización jerárquica del territorio, afirmación que no puede ser probada, mientras no se lleve a cabo una precisa clasificación cultural, cronológica y funcional de cada uno de los yacimientos.

De todo lo anteriormente expuesto se deduce que la elección del lugar para el emplazamiento viene determinada por:

- Las características físicas del lugar. Siempre se eligen "sitios" con abundantes afloramientos rocosos, en alturas que oscilan entre los 740 y $850 \mathrm{~m}$. sobre el nivel del mar, lo que hace factible un ahorro considerable de tiempo y energía en la construcción de las defensas del yacimiento.

- Una distribución organizada de los asentamientos. La elección del lugar y la distribución de los yacimientos parecen estar marcados por unos modelos prefijados con anterioridad a la ocupación del territorio (asentamientos en cima, collado y ladera).

- Las posibilidades de acceso a los recursos. Aunque todos los yacimientos se asientan en lugares de fuertes pendientes, existe, al menos, una zona por la que se accede fácilmente a los valles y vaguadas, factor que nos hace suponer que hay una relación directa entre la función y localización del yacimiento (Fernández, 1984).

- Proximidad a nacimientos de agua. Una característica que define a todos estos asentamientos es la proximidad a pozos o aljibes, que permiten un fácil suministro de agua (Hierro, 1973). Estos aljibes se localizan, indistintamente, en el interior o exterior de los poblados, apareciendo, por lo común, entre las fallas de la cuareita y conservan, generalmente, el agua durante todo o gran parte del año. En algunos de estos affibes aún se pueden apreciar los trabajos de acondicionamiento (excavación, cierre con muros de mampostería, revocos, preparación de la roca, etc.) llevados a cabo para un mejor aprovechamiento de las aguas subterráneas.

Resulta difícil y problemático llevar a cabo un estudio detallado de cada uno de los yacimientos (niveles semimicro y microespacial), partiendo de la sola base del análisis de los restos perceptibles a nivel superficial. En algunos casos la vegetación, la erosión, la acción antrópica o el simple paso del tiempo han destruido o borrado las huellas del pasado, habiendo quedado reducidas a informes amontonamientos de piedra. En otros quedan, todavía, los vestigios suficientes para esbozar y comprender el panorama urbanístico del yacimiento.

La reconstrucción de la casa característica de estos asentamientos no resulta fácil de llevar a cabo, aunque todos los indicios detectados en los sucesivos trabajos de campo nos permiten equipararlas a las documentadas en las excavaciones del cerro de la Encantada (Nieto y Sánchez, 1980). En el yacimiento $n^{2} 9$ se aprecian en el interior del recinto una serie de estructuras de planta circular, cuya puerta de entrada estaba formada por dos grandes lajas de cuarcita hincadas en el 


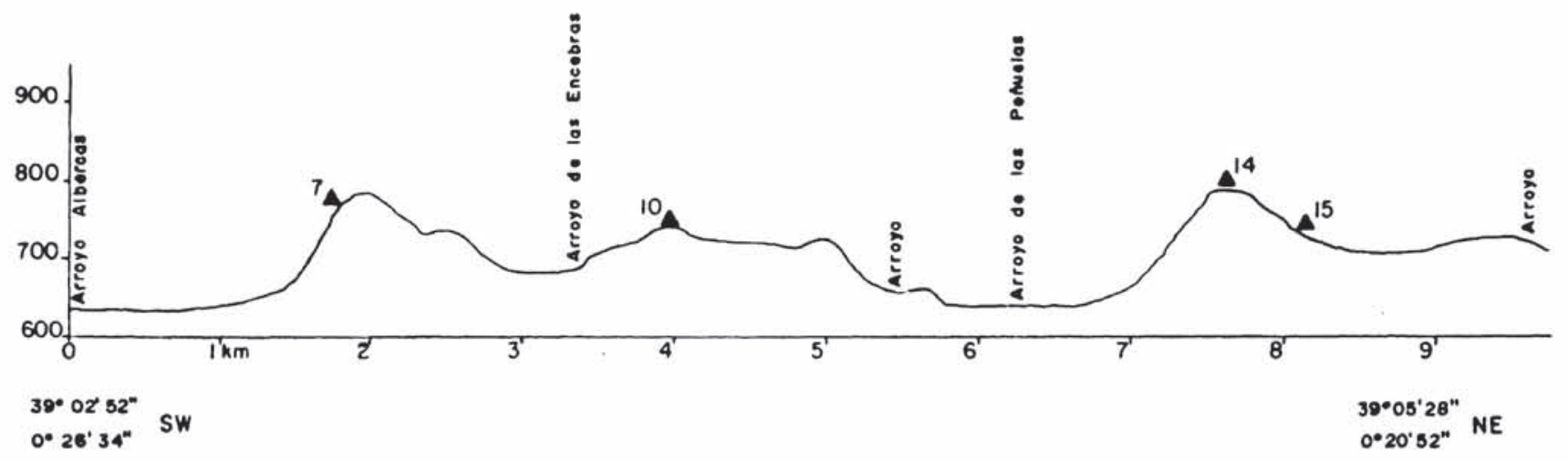

CORTE: A

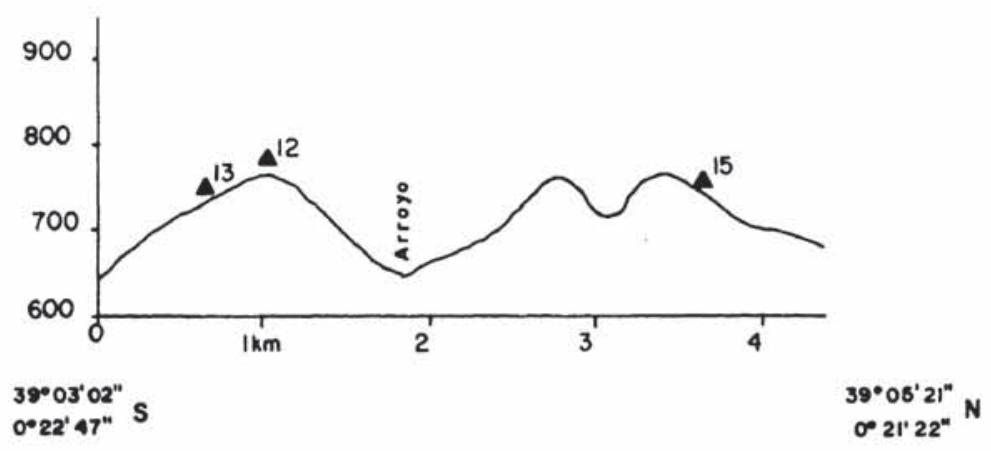

CORTE: $B$

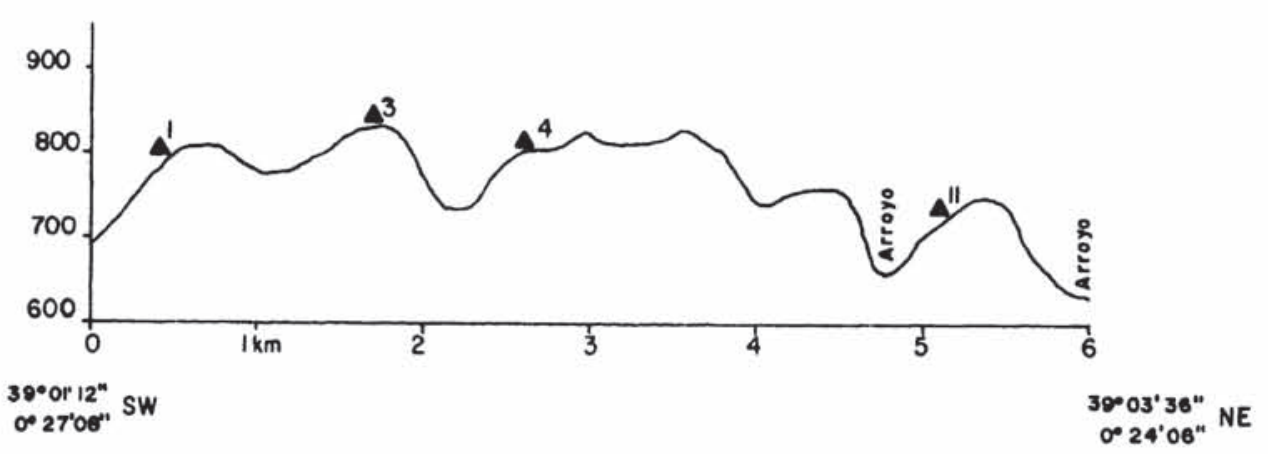

CORTE: :C

- FIG. 3. Cortes Topográficos de las Sierras de Picón y Piedrabuena. 
suelo. Este tipo de vivienda lo volvemos a encontrar, perfectamente definido, en el vacimiento $\mathrm{n}^{2} 14$, aunque aquí aparecen combinadas con otras de planta cuadrada. El mismo tipo de estructura parece adivinarse en el resto de asentamientos, aunque no están claramente definidas por hallarse completamente derruidas y estar reducidas a ingentes amontonamientos de piedras.

La parte de los muros que se conserva es de mampostería de piedras de diverso tamaño, pudiendo estar o no, trabadas con barro. Este sistema de trabazón está asociado, como ocurre en otros yacimientos, a estructuras de planta cuadrada o rectangular (Nieto y Sánchez, 1980), mientras que las de planta circular no tienen trabazón alguna.

El alzado de estas casas debia ser de tierra apisonada (tapial), entre la que se entremezclarían algunas piedras, como así parecen indicarlo los restos de piedras y tierra encontrados en el interior de algunos de estos asentamientos. No cabe descartar, tampoco, la existencia de cabañas fabricadas con materias vegetales (Romero, 1986), especialmente en aquellas construcciones utilizadas como habitación temporal.

Se han documentado algunas covachas cerradas por muros de mampostería, utilizadas, posiblemente, para enterramientos colectivos, de características similares a las descritas por otros autores para la zona de los Montes de Toledo (Nájera, 1984).

Las defensas del recinto de habitación estaban constituidas, en la mayoría de los casos, por las paredes de roca, en cuyos espacios libres se colocaron tramos de muro (piedras sin trabar). Cuando no existen estos farallones rocosos se amuralla todo el recinto.

Estos sistemas defensivos ofrecen ligeras variaciones entre sí. El método más sencillo consiste en fortificar todo el perímetro habitado, reforzando, a modo de acrópolis, la zona más elevada (n.오 1,3 , $5,7,8,9,10,11$ y 15). El segundo sistema consta de varios recintos amurallados y acrópolis (n. os 4 y 12). En tercer lugar aparecen unas defensas complejas, formadas por varias líneas de muralla flanqueadas por torres (n.으 6 y 14).

La adaptación de los sistemas defensivos al terreno impide la aplicación de modelos constructivos preconcebidos, aunque en la mayoría de los casos se percibe la utilización de unos esquemas arquitectónicos fijos (murallas, torreones, acrópolis, etc.), coincidiendo, generalmente, con aquellos lugares que menos defensas naturales poseen.

Todos los asentamientos localizados tienen una segunda fortificación (acrópolis) sobre el lugar más elevado del emplazamiento, en ocasiones por su reducido tamaño puede tratarse, simplemente, de una torre de vigilancia o estructura similar.

Las dimensiones de estos recintos son variables, pudiéndose apreciar, de visu, las diferencias existentes entre ellos. La valoración que haremos del tamaño de los mismos es meramente aproximativa, teniendo en cuenta que, por falta de medios, no se llevaron a cabo levantamientos topográficos y que las mediciones se efectuaron con una cinta métrica y en ocasiones contando pasos. El análisis de los resultados obtenidos nos ha permitido diferenciar cuatro grupos:

- Pequeños asentamientos. No superan los $1.500 \mathrm{~ms}^{2}$; se trata del grupo de asentamientos que anteriormente definimos como de ladera (n.. s 2, 5, 7, 11 y 15). Su reducido tamaño y los escasos restos de estructuras interiores nos llevan a pensar en la posibilidad de que estos yacimientos tuvieran un carácter meramente temporal.

- Asentamientos medianos. Tienen una extensión variable, entre 1.600 y $4.500 \mathrm{~ms}^{2}$; se trata de los yacimientos localizados en los collados y algunos de las cabeceras de sierra (n.os 1, 4, 6 y 9). Este grupo parece tener una actividad relacionada con el control de pasos de montaña y cañadas ganaderas.

- Grandes asentamientos. Tienen una extensión que oscila entre los 5.000 y $10.000 \mathrm{~ms}^{2}$; estos lugares se localizan sobre las cotas de mayor altura (n.os 3 y 10), gozando de un gran control del territorio.

- Yacimientos excepcionales. Alcanzan una extensión que supera los $10.000 \mathrm{~ms}^{2}$ (n.s 8, 12, y 
14). Se localizan en cerros con amplias mesetas (en la tradición local se les conoce como "viviendas de morosn); aunque no se ubican sobre grandes alturas tienen un buen control del territorio, al mismo tiempo que gozan de buena disposición para el establecimiento de relaciones visuales con el resto de yacimientos.

Sin duda el gran problema que se suscita a la hora de analizar el territorio de explotación de estos asentamientos es la carencia de trabajos científicos que pongan en relación los caracteres edáficos existentes en la actualidad con los del II milenio a. C., por lo que el estudio de los rasgos edafológicos actuales nos puede ayudar a comprender los de milenios anteriores.

En la zona objeto de nuestro estudio encontramos terrenos aluviales que se localizan en el fondo de los valles de los ríos y en los arroyos de mayor entidad. Son suelos de excelente fertilidad, siendo muy aptos para el aprovechamiento agrícola (Fig. 4), pues permiten cultivos intensivos y de huerta. Los yacimientos n.․․ 3, 12, 13, 14 y 15 están directamente relacionados con ellos.

Los suelos Pardos Meridionales se localizan en las grandes pendientes, extendiéndose por las laderas de las sierras sobre las que se ubican los asentamientos (Fig. 4). Su aprovechamiento es para pastizal con encinas, alcornoques y jarales. Las superficies con horizontes más desarrollados permiten el cultivo de cereal y/o el olivar.

Los suelos Rojos Mediterráneos se encuentran distribuidos de manera aleatoria por todo el territorio, especialmente en las pendientes suaves, en las que pueden sobresalir crestas de cuarcita no erosionadas (Fig. 4). Tienen un rendimiento aceptable en cultivos como cereal, vid y olivar.

Calibrar y definir el territorio de explotación de estos yacimientos es una tarea ardua y difícil, pues carecemos de datos objetivos referentes a la actividad de estas poblaciones, por lo que no entraremos a delimitar con exactitud cual era el espacio real de cada uno de los asentamientos, en espera de que los resultados de futuras investigaciones nos permitan establecer tales valoraciones; no obstante, presentamos, a título meramente indicativo, una tabla con los datos obtenidos de las calibraciones realizadas para radios de $5 \mathrm{kms}$.

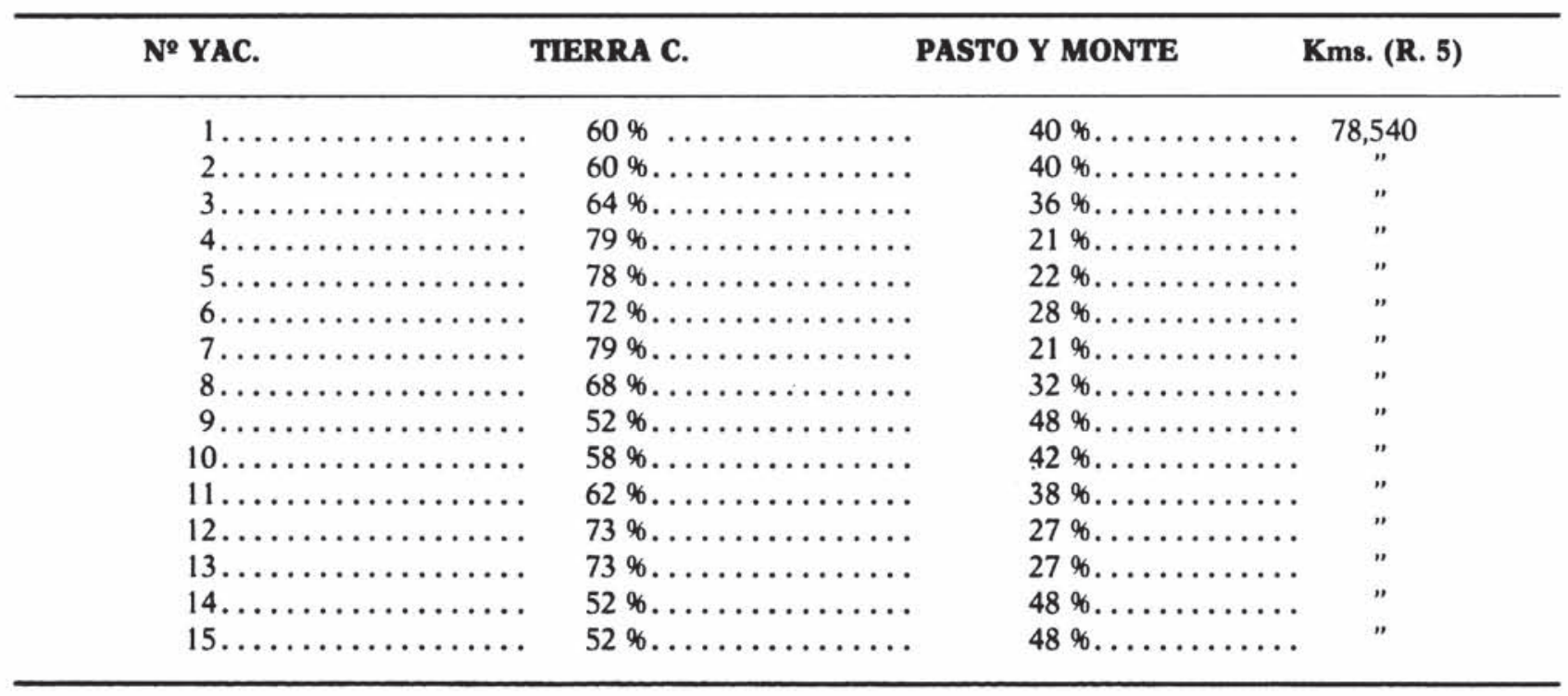

En esta tabila queda reflejado el gran potencial de explotación agrícola de algunos yacimientos (n.os 3, 4, 5, 6, 7, 8, 12 y 13), frente a otros que presentan una posición de dominio de unos terrenos más favorables a la explotación ganadera (n.오 1, 2, 9, 10, 11, 14 y 15). De esta evaluación se deduce que el número de yacimientos es equiparable desde el punto de vista de las posibilidades que ofrece 


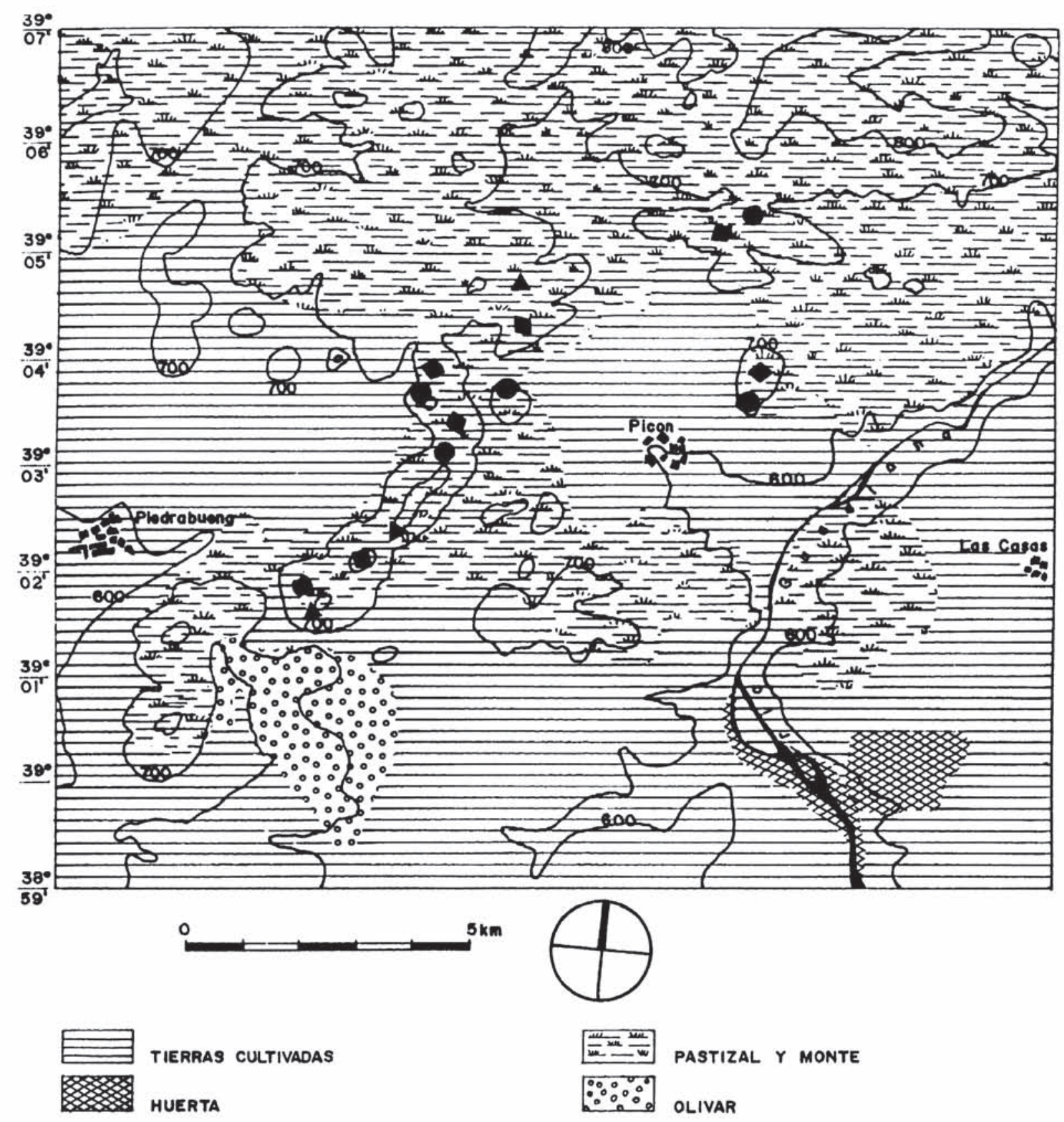

Fig. 4. Territorio de Explotación.

el medio, lo que no significa que esto fuese necesariamente así, pues cabe la posibilidad que alguno de los asentamientos mencionados tuviera otra $u$ otras actividades predominantes (vigilancia, caza, comercio, industrias de transformación, etc.).

El territorio, a nuestro modo de ver, estaría controlado por los yacimientos de mayor tamaño (3, $4,8,10,12$ y 14), funcionando el resto como aledaños o pedanías de estos, hipótesis que se encuentra en fase de confrontación en base a los trabajos y mediciones que estamos llevando a cabo en zonas próximas, especialmente en la franja sur de los Montes de Toledo.

Uno de los aspectos fundamentales, a nuestro juicio, del trabajo del campo consiste en la recogida de materiales. De su estudio y posterior análisis se puede llegar a deducir una cronología y 
una clasificación cultural, sino exactas, al menos aproximadas. Desgraciadamente los materiales recogidos en la zona son escasamente representativos. En primer lugar por ser fragmentos de reducido tamaño, que apenas son orientables y en segundo lugar por tratarse de materiales sometidos a un largo proceso de rodamiento, habiendo perdido parte de la textura original. Se recogieron dos tipos de materiales, cerámicos y líticos, reflejados en la siguiente tabla:

\begin{tabular}{|c|c|c|c|c|c|}
\hline & NOMBRE & BORDES & GALBOS & LITICo & TOTAL \\
\hline 1) & Morro del Sotajo & 00 & 00 & 00 & 00 \\
\hline 2) & Morro Pinto & 00 & 01 & 00 & 01 \\
\hline 3) & Cerro Benavente & 05 & 35 & $\mathbf{0 0}$ & 40 \\
\hline 4) & Collado los Criminales & 09 & 38 & 02 & 49 \\
\hline 5) & Hoya del Lobo & 00 & 01 & 00 & 01 \\
\hline 6) & Aljibe la Vieja & 00 & 03 & 00 & 03 \\
\hline 7) & Morro la Dueña & 03 & 07 & 00 & 10 \\
\hline 8) & Aljibe del Moro & 09 & 32 & 00 & 41 \\
\hline 9) & Cerro Cañejares & 01 & 03 & 00 & 04 \\
\hline 10) & Cerro del Tesoro & 01 & 17 & 01 & 19 \\
\hline 11) & Cerro Majadaciemente & 00 & 00 & 00 & 00 \\
\hline 12) & Plaza de los Moros & 04 & 38 & 00 & 42 \\
\hline 13) & Cerro de la Contera & 00 & 00 & 00 & 00 \\
\hline 14) & Cerro del Acebuchar I & 00 & 02 & 00 & 02 \\
\hline \multirow[t]{2}{*}{ 15) } & Cerro del Acebuchar II & 00 & 00 & 00 & 00 \\
\hline & TOTAL & 32 & 187 & 03 & 212 \\
\hline
\end{tabular}

Las cerámicas constituyen el grueso del material recogido en la prospección, sin embargo ninguno de los fragmentos tiene un tamaño que permita una identificación formal segura, por lo que resulta imposible llegar a establecer paralelos tipológicos y tipométricos con otros yacimientos.

Las formas que aparecen en los yacimientos del área de Picón y Piedrabuena son las típicas de la Edad del Bronce, predominando cuencos, ollas y vasos carenados (Fig. 5), pero todo ello tratado con las reservas que impone el trabajar con fragmentos de reducido tamaño, pues en algunos casos no es posible ni una identificación formal aproximada.

Estas cerámicas se caracterizan por tener las pastas y superficies claras y medias (tonos rojizos, anaranjados y ocres), cocción oxidante (66,67 \%); frente a las oscuras (tonos grises y negros), cocción reductora $(33,33 \%)$.

El análisis del acabado de estas cerámicas es bastante problemático, pues todas ellas han estado sometidas a un fuerte rodamiento, aunque de su estudio hemos podido extraer los siguientes datos estadísticos, donde queda reflejado el claro predominio de los alisados $(63,51 \%)$ frente al resto; sin embargo, el bajo porcentaje de espatulados $(17,13 \%)$ y bruñidos $(9 \%)$ puede ser debido al mal estado de conservación (grosero 10,36\%), pues se trata de materiales de superficie, expuestos continuamente a las inclemencias del tiempo.

Del estudio global de la cerámica se deduce que las formas y los acabados son paralelizables a los del Cerro de la Encantada, donde aparecen ollas, cuencos y vasos carenados de pequeño y mediano tamaño (Nieto y Sánchez, 1980), alisados, espatulados y bruñidos. También existe un cierto parecido con alguno de los materiales de las motillas (Nájera, 1977; Colmenarejo, 1987).

El material lítico está escasamente representado en las muestras recogidas. Se trata de dos lascas de sílex sin retoque y un posible botón de caliza, de dudosa clasificación. 
POBLADOS DE ALTURA DE LA EDAD DEL BRONCE EN LAS SIERRAS DE...
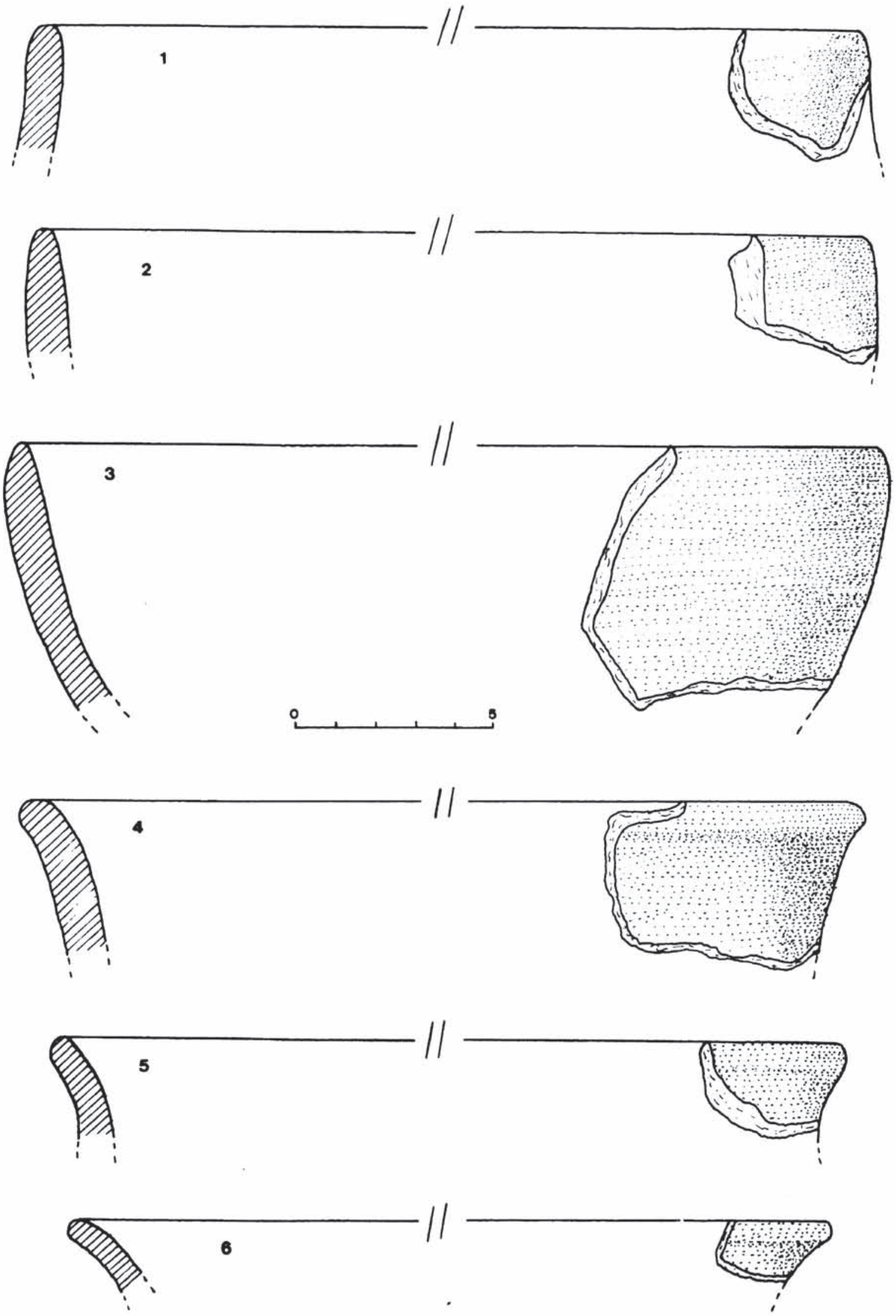

FIG. 5. Cerámica (1 y 2, Yacimiento $n^{\circ}$ 7; 3, 4, 5 y 6 Yacimiento $\left.n^{\circ} 8\right)$.

T. P., $1990, \mathrm{n}^{2} 47$ 
Examinando los resultados obtenidos en los análisis del tipo de poblamiento y materiales arqueológicos, creemos que los yacimientos del área de Picón y Piedrabuena deben ser adscritos a la Edad del Bronce, sin que por el momento podamos precisar a cual de sus fases pertenecen, debido a la inexistencia de excavaciones sistemáticas en la zona.

Antes de terminar quisiéramos aclarar aquellas interpretaciones que pudieran derivarse del presente trabajo, tales como la ausencia de asentamientos en llano. Conocemos la existencia de este tipo de asentamientos en la zona, aunque todavia no tenemos definidas sus principales características por tratarse de hallazgos esporádicos poco sistematizados. De todos modos no hemos creído oportuno incluirlos en el presente estudio hasta que no dispongamos de datos suficientemente confirmados y confrontados con los que estamos obteniendo en zonas próximas.

\section{BIBLIOGRAFIA}

'Caballero Klink, A. y otros (1983): Catálogo de Bibliografia Arqueológica de la provincia de Ciudad Real. Museo de Ciudad Real. Estudios y Monografías, 10. Ciudad Real.

Colmenarejo Hernández, R. y otros (1987): "La Motilla de Sta. María del Retaman. Oretum III: 79-109. Ciudad real.

CHISHOLM, M. (1968): Rural settlement and land use: an essay in location. Londres. 2.a edición.

FernÁNDEZ MARTíneZ, V. y Ruiz ZaPATERo, G. (1984): «El análisis de territorios arqueológicos: una introducción crítica*. Actas del I Congreso de Arqueología Espacial I: 55-73. Teruel.

Hierro del. Real, L. (1973): "Los Castellones de Picón (Ciudad Real)». Cuadernos de Estudios Manchegos, 4, 2. ${ }^{a}$ época: 111-119. Ciudad Real.

LEE, R. B. (1969): «I Kung Bushman Subsistence: an input-output analysis»; en A. P. VAYDA (ed.). Environment and cultural Behaviour: 47-79. New York.

López FernÁndez, F. J. (1988): «La Edad del Bronce en las estribaciones Meridionales de los Montes de Toledo (Ciudad Real)n. Actas del I Congreso de Historia de Castilla. La Mancha, II: 283-290. Ciudad Real (1985). Ciudad Real.

Miranda, J. M. y Sánchez Meseguer, J. (1986): «Bases para el estudio de las relaciones entre el medio geográfico y los asentamientos humanos". Actas del II Congreso de Arqueología Espacial, VII: 199-212. Teruel.

Nájera Colino, T. (1984): La edad del Bronce en la Mancha Occidental. Tesis doctorales de la Universidad de Granada, 458. Granada.

Nájera, T. y Molina, F. (1977): «La Edad del Bronce en la Mancha. Excavaciones en las Motillas del Azuer y los Palacios (campaña 1974)». Cuadernos de Prehistoria de la Universidad de Granada, 2: 251-300. Granada.

Nájera, T.; Molina, F. y otros (1977): «Excavaciones en las Motillas del Azuer y los Palacios (Daimiel, C. Real). Actas del XIV Congreso Nacional de Arqueologia: 503-514. Zaragoza.

- (1979): «La Motilla del Azuer (Daimiel, C. Real)». Noticiario Arqueológico Hispánico, VI: 19-50. Madrid.

Nieto Gallo, G. y Sánchez Meseguer, J. (1980): El Cerro de la Encantada. Excavaciones Arqueológicas en España, 113. Madrid.

Nieto Gallo, G.; Sánchez Meseguer, J. y otros (1983): «El Cerro de la Encantada (Granátula de Cva., Ciudad Real)». Noticiario Arqueológico Hispánico, XVII: 7-42. Madrid.

Romero Salas, H.; Cantero Desmartines, M. C. y Martínez Peñarroya, J. (1986): «Estructuras de habitación del Bronce de la Mancha: la primera fase de ocupación del cerro del Cucon. Actas del II Congreso de Arqueología Espacial, VIII: 235-246. Teruel.

Viñas, C. y PAZ, R. (1971): Relaciones de los Pueblos de España ordenadas por Felipe II: Ciudad Real 1975. Madrid. 